\title{
Atypical cellular blue nevus or malignant blue nevus?*
}

\author{
Luise Ribeiro Daltro ${ }^{1}$ \\ Rodrigo Abdalah Freitas ${ }^{2}$ \\ Cacilda da Silva Souza ${ }^{1}$
}

\author{
Lygia Bertalha Yaegashi ${ }^{2}$ \\ Bruno de Carvalho Fantini ${ }^{1}$
}

DOI: http:/ / dx.doi.org/10.1590/abd1806-4841.20174502

\begin{abstract}
Blue nevus is a benign melanocytic lesion whose most frequent variants are dendritic (common) blue nevus and cellular blue nevus. Atypical cellular blue nevus presents an intermediate histopathology between the typical and a rare variant of malignant blue nevus/melanoma arising in a cellular blue nevus. An 8-year-old child presented a pigmented lesion in the buttock since birth, but with progressive growth in the last two years. After surgical excision, histopathological examination revealed atypical cellular blue nevus. Presence of mitoses, ulceration, infiltration, cytological atypia or necrosis may occur in atypical cellular blue nevus, making it difficult to differentiate it from melanoma. The growth of blue nevus is unusual and considered of high-risk for malignancy, being an indicator for complete resection and periodic follow-up of these patients.
\end{abstract}

Keywords: Melanoma; Nevus, blue; Nevus, pigmented

\section{INTRODUCTION}

Blue nevus $(\mathrm{BN})$ and related entities are a heterogeneous group of congenital and acquired melanocytic tumors that includes dendritic ("common") blue nevus (DBN), cellular blue nevus (CBN), and variants, as atypical cellular blue nevus (ACBN) and malignant BN/melanoma. ${ }^{1,2}$ They share several clinical and morphologic features including their blue tinctorial properties; dermal proliferation of spindle, fusiform or ovoid cells; melanin pigment inside both the melanocytic tumor cells and macrophages; and variably stromal fibrosis and collagen amounts. Immunophenotypically, the tumors have positivity for HMB-45 (Gp100), S-100 and Melan-A/Mart-1 expression. ${ }^{1,2,3}$

CBN differs from the classic DBN by its large size, cellularity, intense pigmentation, and growing pattern with subcutaneous infiltration, but with variable overlapping. Additional atypical features associated with variety of CBN histological patterns, but without clear-cut evidence of malignancy, has been referred to as ACBN, and it can be difficult to it distinguish from melanoma. ${ }^{1,2,3,4}$ Malignant BN is a very rare form of melanoma, arising in association with or exhibiting some morphologic similarities to $\mathrm{BN} .^{1,3,4} \mathrm{In}$ view of its rarity, the relevance and the challenge of differentiating it from its malignant variant, we report a case of congenital BN with progressive growth, histologically consistent with an atypical CBN.

\section{CASE REPORT}

An 8-year-old boy was presented with a dark lesion on buttock, since birth, but with a progressive growth in two years. Physical examination revealed a dark blue nodule with homogeneous pigmentation, symmetrical and regular borders, with $2.5 \mathrm{~cm}$ in diameter on the left gluteus, with no palpable lymph nodes (Figure 1A). Dermoscopy revealed a homogeneous and structureless pigment pattern of grayish-brown color, and a slightly irregular surface (Figure 1B). Surgical excision was performed with $5 \mathrm{~mm}$ margin due to the difficulty in delimiting the lesion. Histological examination revealed a solid melanocytic lesion, composed by hypercellular nests affecting the entire dermis and part of subcutaneous tissue (Figure 2). In the nest extremity, there were dendritic pigmented cells; in the center, ovoid and pale cells, with intermediate size; and focally, pleomorphic and vesicular nuclei, evident nucleoli, and occasional mitosis $<2 / \mathrm{mm}^{2}$, which was compatible with ACBN (Figures 3 and 4).

\section{DISCUSSION}

BN usually appears in childhood or adolescence and is rarely congenital. ${ }^{1,2}$ The most common form, DBN, is presented as a solitary, symmetrical and intensely pigmented papule or nodule measuring less than $1 \mathrm{~cm}$ and occurs mainly on the back of the hands or feet; or locations as face and scalp. ${ }^{1,2}$ It can affect mucous mem-

Received on 24.02.2015.

Approved by the Advisory Board and accepted for publication on 13.01.2016.

* Study conducted at Hospital das Clínicas da Faculdade de Medicina de Ribeirão Preto- Universidade de São Paulo (FMRP-USP) - Ribeirão Preto Financial Support: None.

Conflict of Interest: None.

Division of Dermatology, Internal Medicine Department - Medical School of Ribeirão Preto - Universidade de São Paulo- Ribeirão Preto (SP) -Brazil. Department of Pathology - Medical School of Ribeirão Preto - Universidade de São Paulo- Ribeirão Preto (SP) -Brazil. 

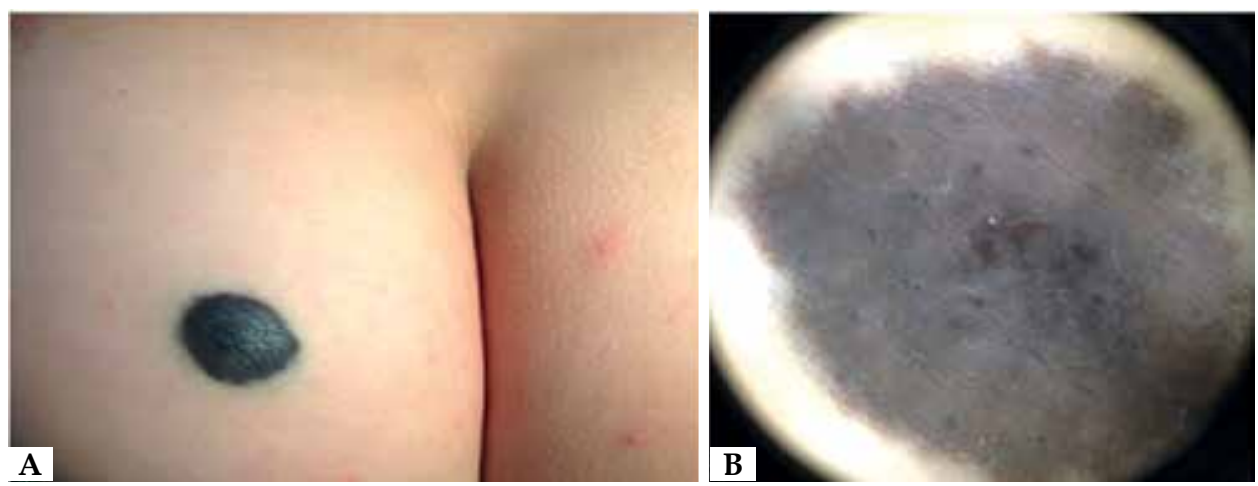

FiguRe 1:

A) Nodule with dark blue homogeneous pigmentation, symmetrical and regular borders, measuring 2.5 $\mathrm{cm}$ in diameter on gluteus; B) Dermoscopy exhibited a homogeneous and structureless pigment pattern of grayish-brown color, more evident in the follicular ostia

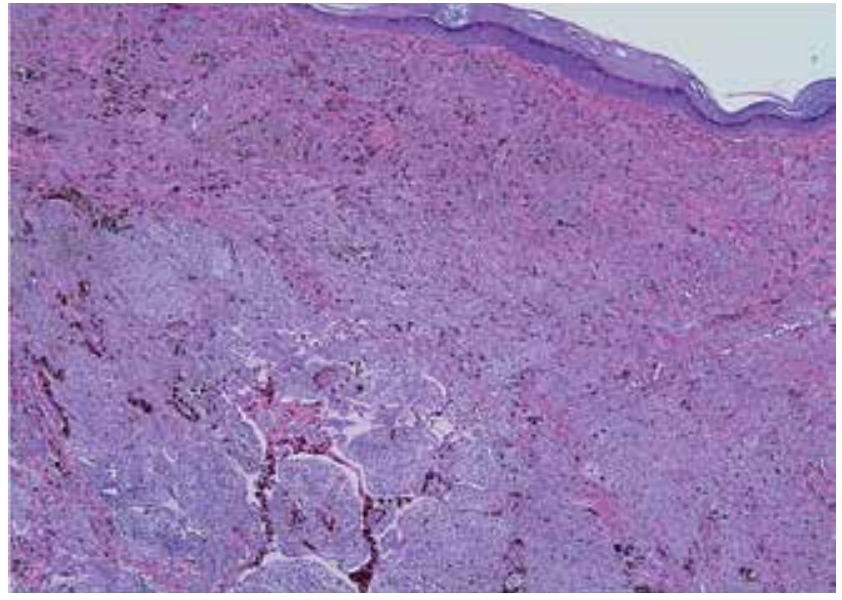

FIGURE 2: Melanocyte proliferation occupying papillar and reticular dermis, and forming nests; no alteration in the epidermis was observed (Hematoxylin \& eosin $x 40$ )

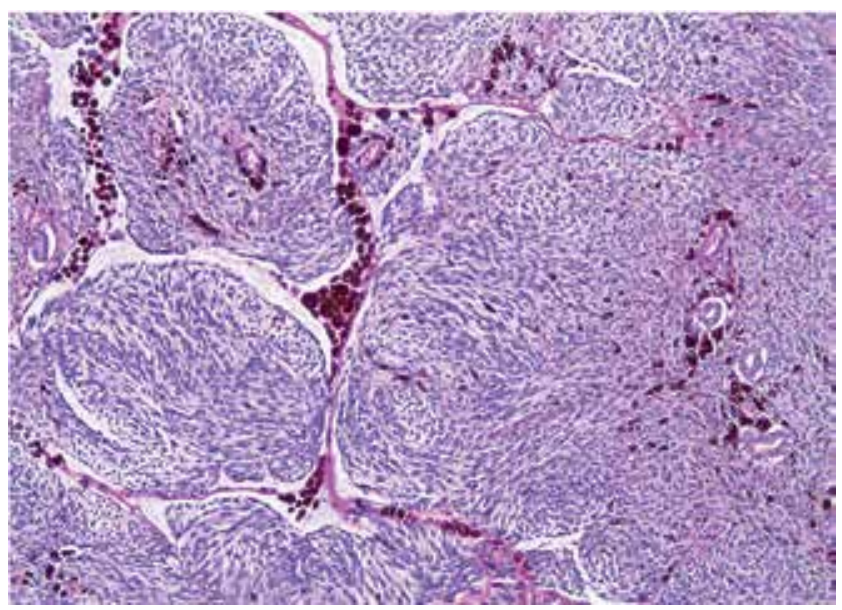

Figure 3: Nests compose of heavily pigmented and dendritic melanocytes in the periphery; pale and ovoid melanocytes of intermediate size in the center were consistent with cellular blue nevus (Hematoxylin \& eosin $x 100$ )

branes and extracutaneous sites. CBN variant is a nodule or plaque, usually 1 to $3 \mathrm{~cm}$ in diameter, with smooth or irregular surface, most commonly found in the glutei, sacrococcygeal area or scalp., ${ }^{1,2}$ Growth is unusual and should raise suspicion of malignant degeneration. ${ }^{1,2,4,5}$

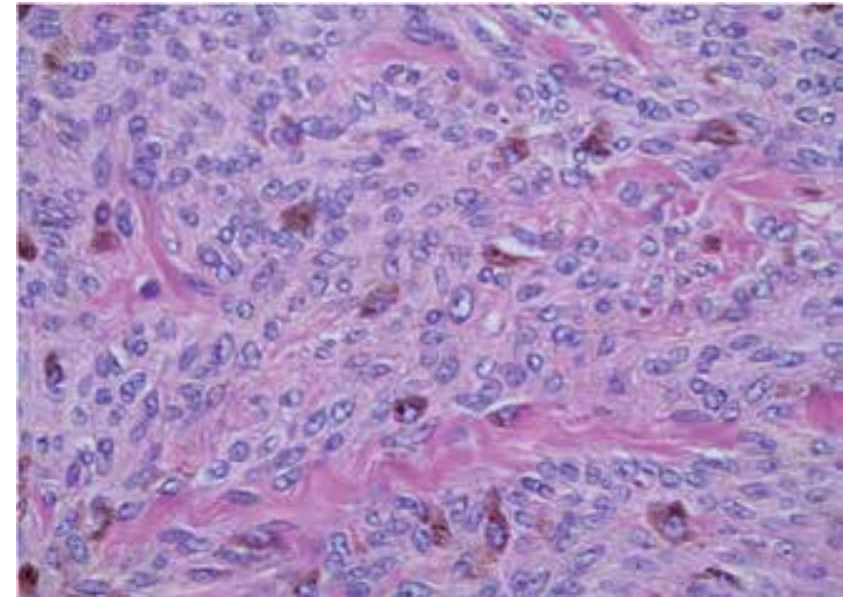

FIGURE 4: Pleomorphic and vesicular nuclei, evident nucleoli, and occasional mitosis $\left(<2 / \mathrm{mm}^{2}\right)$ were compatible with atypical cellular blue nevus (Hematoxylin \& eosin $x 400$ )

Malignant BN has usually a multinodular appearance, with progressive growth and, in general, more than $3 \mathrm{~cm}$ in diameter, and located on the scalp. ${ }^{1}$ It can arise from a $\mathrm{BN}$, nevus of Ota or Ito or de novo, and its biological behavior is uncertain. ${ }^{1,2,4,5}$ Malignant degeneration has unknown incidence and prognosis, with a high rate of recurrence and metastasis, especially to lymph nodes. ${ }^{1,2,4,5}$ High risk characteristics for malignancy in a $\mathrm{BN}$ are: size $>2 \mathrm{~cm}$, multinodular surface, location in scalp and growth. ${ }^{1,2,4,5,6} \mathrm{It}$ is believed that $\mathrm{BN}$ derives from an incomplete migration of melanocytes precursor cells, coming from the neural crest, on its way to the epidermis. Histologically, there are dendritic melanocytes, grouped in the upper and middle dermis, with active melanin production, whereas in compound and intradermal nevi, dermal melanocytes do not produce, or produce little, melanin. ${ }^{1}$

CBN differentiates from DBN by pigmented melanocytes in the dermis, grouped spindle cells containing little or none melanin, that frequently penetrate the subcutaneous layer as well-defined islands. ${ }^{1,2,3,4,5,6}$ Although, there is no uniform criteria for the distinction, high mitotic activity $(>2 / \mathrm{mm} 2)$, marked cytological atypia, necrosis, deep infiltration and atypical mitotic figures favor melanoma. ${ }^{1,2,3,4,5,6}$ At least one of the following findings characterizes CBN as atypical: asymmetry, focal infiltration or hypercellularity, pleomorphism with hyperchromasia, proeminent nucleoli, and occasional mitoses $\left(<2 / \mathrm{mm}^{2}\right)$. ${ }^{1,2,3,4,5,6}$

Because of the overlapping intermediate features between 
atypical $\mathrm{CBN}$ and malignant $\mathrm{BN} /$ melanoma, its distinction remains a challenge. ${ }^{5,7} \mathrm{ACBNs}$ tend to show a higher proliferative index by proliferating cell nuclear antigen (PCNA) and Mib-1 as well as a significantly higher mitotic rate compared with typical CBN.

Argyrophilic nucleolar organizer regions (AgNORs) expression has been considered in skin melanocytic lesion prognosis. AgNOR mean number per nucleus (AgNOR count), their distribution (configuration) and the ratio AgNOR area/nuclear area can be useful to discriminate benign from malignant melanocytic lesions, although with considerable overlap. Usually, there is a linear relation between AgNOR counts and PCNA immunostaining scores, which can be additional parameters for the diagnosis of malignant CBN. ${ }^{7}$

Molecular techniques of the primary tumor such as comparative genomic hybridization (CGH) or fluorescence in situ hybridization may assist in establishing a melanoma diagnosis. However, these tests require further independent validation and are not widely available at present. ${ }^{8}$

CGH of some cases of large plaque-type BN with subcutaneous cellular nodules have showed chromosomal aberrations typical of melanoma. Nodules showed gains involving chromosome $6 \mathrm{p}$ and losses involving chromosome 6q, which are among the most commonly found aberrations in melanoma. In the less cellular surrounding areas that appeared $\mathrm{BN}$ representative, these copy number aberrations were not present. ${ }^{9}$

Large BN with a deep, multi-nodular configuration should be interpreted with caution, and superficial biopsy can be misleading. Patients with histologically ambiguous dermal melanocytic proliferations that exhibit copy number aberrations should undergo careful clinical follow-up..$^{10}$

Somatic mutations in codon 209 of GNAQ, a gene encoding the signaling protein G-protein $\alpha$ subunit $q$, may be present in up to $80 \%$ of $\mathrm{BN} .^{10}$

The difficult distinction of ACBN from melanoma associated with growth, a high-risk malignancy characteristic, but unusual in CBN, justify its complete resection and long-term follow-up of these patients. Further studies including molecular and genetic analysis will provide valuable insights and may be useful in differentiating atypical CBN from malignant $\mathrm{BN}$, but the accurate history and physical examination, with attention to the lesion growth, remain fundamental for early intervention.]

\section{REFERENCES}

1. Murali R, McCarthy SW, Scolyer RA. Blue nevi and related lesions: a review highlighting atypical and newly described variants, distinguishing features and diagnostic pitfalls. Adv Anat Pathol. 2009;16:365-82.

2. Barnhill RL, Rabinovitz Harold. Neoplasias melanocíticas benignas. In: Bolognia JL, Jorizzo JL, Rapini RP. Dermatologia. 2. ed. Rio de Janeiro: Elsevier; 2011. p. 1722-23.

3. Zembowicz A, Mihm MC. Dermal dendritic melanocytic proliferations: an update. Histopathology. 2004;45:433-51.

4. Barnhill RL, Argenyi Z, Berwick M, Duray PH, Erickson L, Guitart J, et al. Atypica cellular blue nevi (cellular blue nevi with atypical features): lack of consensus for diagnosis and distinction from cellular blue nevi and malignant melanoma ("malignant blue nevus"). Am J Surg Pathol. 2008;32:36-44.

5. Tran TA, Carlson JA, Basaca PC, Mihm MC. Cellular blue nevus with atypia (atypical cellular blue nevus): a clinicopathologic study of nine cases. J Cutan Pathol. 1998;25:252-8.

6. Barnhill RL, Cerroni L, Cook M, Elder DE, Kerl H, LeBoit PE, et al. State of the art, nomenclature, and points of consensus and controversy concerning benign melanocytic lesions outcome of an international workshop. Adv Anat Pathol. 2010;17:73-90.

7. Pich A, Chiusa $L$, Margaria $E$. Role of the argyrophilic nucleolar organizer regions in tumor detection and prognosis. Cancer Detect Prev. 1995;19:282-91.

8. Scolyer RA, Murali R, McCarthy SW, Thompson JF. Histologically ambiguous ("borderline") primary cutaneous melanocytic tumors: approaches to patient management including the roles of molecular testing and sentinel lymph node biopsy. Arch Pathol Lab Med. 2010;134:1770-7.
9. North JP, Yeh I, McCalmont TH, LeBoit PE. Melanoma ex blue nevus: two cases resembling large plaque-type blue nevus with subcutaneous cellular nodules. J Cutan Pathol. 2012;39:1094-9.

10. Held L, Eigentler TK, Metzler G, Leiter U, Messina JL, Glass LF, et al. Proliferative activity, chromosomal aberrations, and tumor-specific mutations in the differential diagnosis between blue nevi and melanoma. Am J Pathol. 2013;182:640-5.

\author{
MAILING ADDRESS: \\ Cacilda da Silva Souza \\ Hospital das Clínicas, Divisão de Dermatologia \\ Campus Universitário, $s / n$ \\ Monte Alegre \\ 14048-900 - Ribeirão Preto - SP \\ Brazil \\ E-mail: cssouza@fmrp.usp.br
}

How to cite this article: Daltro LR, Yaegashi LB, Freitas RA, Fantini BC, Souza CS. Atypical cellular blue nevus or malignant blue nevus? An Bras Dermatol. 2017;92(1):110-2. 